\title{
Directed forgetting benefits motor sequence encoding
}

\author{
Tobias Tempel ${ }^{1} \cdot$ Christian Frings $^{1}$
}

Published online: 15 October 2015

(C) Psychonomic Society, Inc. 2015

\begin{abstract}
Two experiments investigated directed forgetting of newly learned motor sequences. Concurrently with the list method of directed forgetting, participants successively learned two lists of motor sequences. Each sequence consisted of four consecutive finger movements. After a short distractor task, a recall test was given. Both experiments compared a forget group that was instructed to forget list-1 items with a remember group not receiving a forget instruction. We found that the instruction to forget list 1 enhanced recall of subsequently learned motor sequences. This benefit of directed forgetting occurred independently of costs for list 1 . A mediation analysis showed that the encoding accuracy of list 2 was a mediator of the recall benefit, that is, the more accurate execution of motor sequences of list 2 after receiving a forget instruction for list 1 accounted for better recall of list 2 . Thus, the adaptation of the list method to motor action provided more direct evidence on the effect of directed forgetting on subsequent learning. The results corroborate the assumption of a reset of encoding as a consequence of directed forgetting.
\end{abstract}

Keywords Body movement $\cdot$ Recall $\cdot$ Directed forgetting · Motor learning

Body control involves retrieval of body movements from memory. In many contexts, such as sports or music, quick

Tobias Tempel

tempel@uni-trier.de

1 Department of Psychology, University of Trier, Trier 54286, Germany and accurate movements are required that depend on successfully accessing corresponding memory representations. Performance is hampered when, for example, the wrong body movement is remembered or the sought movement cannot be distinguished from several similar stored movements. Memories can be a burden. When they become activated involuntarily, they can interfere with retrieval processes as well as impair the acquisition of new information.

Memory representations of body movements are susceptible to interference. For example, retrieval of motor sequences can be impaired by retroactive interference (e.g., Panzer, Wilde, \& Shea, 2006; Wohldmann, Healy, \& Bourne, 2008) and retrieval-induced forgetting (Reppa, Worth, Greville, \& Saunders, 2013; Tempel \& Frings, 2013, 2014a, 2014b, 2015; Tempel, Loran, \& Frings, 2015). Moreover, proactive interference has been shown to affect retrieval (e.g., Ascoli \& Schmidt, 1969; Williams, 1971) as well as the acquisition of motor responses (e.g., Brashers-Krug, Shadmehr, \& Bizzi, 1996; Cothros, Köhler, Dickie, Mirsattari, \& Gribble, 2006).

Directed forgetting (i.e., the attempt to intentionally forget previously encoded information) is known to reduce interference during retrieval in different domains. With regard to motor action, a study by Burwitz (1974) on short-term motor memory showed that directed forgetting can reduce proactive interference. After participants had learned several linear lever displacements, an instruction to forget initially learned responses facilitated retrieval of the last lever displacement learned, improving accuracy. Dual-process models (Pastötter \& Bäuml, 2010; Sahakyan \& Delaney, 2003) assume that directed forgetting not only reduces interference during retrieval, but also enhances subsequent encoding. Here, we investigated whether directed forgetting of just-learned motor sequences is also capable of enhancing the subsequent learning of motor sequences. We adapted the list method of directed forgetting for this purpose. The list method has been 
extensively used with verbal materials. Whereas an extension to action phrases (that were either enacted by participants or merely read; Earles \& Kersten, 2002; Sahakyan \& Foster, 2009) already involved encoding of motor components in addition to verbal representations, non-verbal motor sequences have not been used as material before.

The list method compares two groups of participants. Both receive two learning lists (L1 and L2) and a recall test. After $\mathrm{L} 1$, the forget group receives the instruction to forget the thus far presented items. The remember group, on the other hand, is informed that they had just learned the first half of items and would now continue with the second half. In a final test, participants in both groups are then asked to recall the items from both lists. Typically, a benefit of directed forgetting emerges as more L2 items are recalled in the forget group than in the remember group. In addition, often a cost effect emerges, that is, the forget group recalls significantly fewer L1 items.

Reduced proactive interference from the to-be-forgotten L1 (Bjork, 1989) as well as better encoding of L2 items contribute to the benefit effect. According to Sahakyan and Delaney (2003), a forget instruction triggers a change of encoding strategies to deeper encoding of L2 items, whereas participants in a remember group rather maintain strategies they used at encoding L1 when moving on with L2. Pastötter and Bäuml (2010) also assume better encoding of L2 but specify that a reset of encoding may account for the benefit effect. They found that especially the first L2 items benefited from a forget instruction. According to the reset-of-encoding assumption, encoding efficacy decreases with an increase in study material, that is, it decreases from L1 encoding to L2 encoding due to inattentional encoding and increased memory load. A forget cue can abolish memory load and inattentional encoding, and thus make encoding of early L2 items as effective as the encoding of early L1 items. In addition, Pastötter, Kliegl, and Bäuml (2012) demonstrated that the testing order impacted the benefit effect. Only with L1 tested before L2, the benefit effect was restricted to early L2 items, suggesting that better encoding of L2 accounted for the benefit. When L2 was tested before L1, there was an additional benefit for all L2 items irrespective of study position. The recall of L2 items apparently profits from better encoding of L2 as well as from the lower accessibility of L1 in the forget group if L2 is tested before L1. Correspondingly, a meta-analysis of directed-forgetting studies showed that the size of benefit effects depends on testing order (Pastötter et al., 2012). When L2 is tested first the benefit is stronger as compared to when L1 is tested first. This pattern matches the assumption that lower interference by L1 as well as better encoding of L2 contribute to the benefit effect when L2 is tested first but only better encoding of L2 remains as an enhancing influence when L1 is tested first (because interference by L1 dissolves after its testing).
Research on directed forgetting has not often focused on motor memory. We examined whether directed forgetting enhances the encoding of motor sequences. Whereas it has already been demonstrated that directed forgetting can reduce interference of the retrieval of motor responses (Burwitz, 1974), we focused on encoding benefits that occur subsequent to a forget instruction.

\section{Experiment 1}

Participants learned sequential finger movements (SFMs) all performed with three fingers of the right hand. Each SFM consisted of four consecutive key presses. According to the list method, two groups were contrasted. After learning L1 comprising five SFMs, the forget group received a forget instruction for this list, whereas the remember group did not receive a forget instruction. Subsequently, L2, also comprising five SFMs, was learned. Items were repeated in 12 learning blocks in both lists. After an unrelated distractor task, participants then had to recall SFMs of both lists. The number of correctly recalled SFMs was analyzed. Following Pastötter et al. (2012), participants had to recall L1 before L2 because this testing order should entail a benefit effect resulting from better encoding while precluding reduced interference to contribute to the benefit. We assumed the intention to forget L1 enhanced subsequent encoding. Hence, we expected superior recall of $\mathrm{L} 2$ in the forget group as compared to the remember group.

\section{Method}

Participants Sixty undergraduate students at the University of Trier participated in the experiment. They either received course credit for their participation or were paid four Euro.

Design Thirty participants each were randomly assigned to one of two groups. The forget group received a forget instruction after learning a first set of SFMs, whereas the remember group did not receive a forget instruction.

Materials The experiment was conducted using Dell Optiplex 755 PCs with Eizo FlexScan S1901 monitors and standard German QWERTZ keyboards. The software PXLab (Irtel, 2007) served for running the experiment.

All ten items consisted of four consecutive finger movements (see Appendix) and were performed with fingers of the right hand. The index, middle, and ring fingers were placed on the keys $V, B$, and $N$, respectively. Labels hiding the inscription marked the keys. During the learning phases an animation of the corresponding SFM appeared on the computer screen. A display of the right hand demonstrated which fingers were to be moved by showing four 
consecutively flashing fingers (the first and third fingers were colored yellow, second and fourth fingers were colored blue, $200 \mathrm{~ms}$ per flash). After the right hand display disappeared participants could perform the movement (cf. Fig. 1, upper section). If the performed sequence was incorrect feedback appeared in the form of the word: "Fehler!" (English: “Error!”).

Procedure The experiment consisted of four phases (learning L1, learning L2, distractor, recall test). Instructions were given on the screen. Participants were told that five SFMs were to be learned in repeated learning blocks and informed about the graphic animation demonstrating the SFM as well as about the response keys. After having read the initial instructions for the learning phase the participant clicked an on-screen button to start with L1. Participants had to consecutively press four keys in response to an animation showing which digits were to be moved. The items were repeated in 12 blocks each presenting the items in a random order. Subsequently, the forget group was instructed to forget the SFMs learned so far because they only served the purpose of warming up and familiarization with the format of presentation. Participants were asked to retain only those SFMs that were going to be practiced subsequently. In contrast, the remember group was informed that they had finished the first part of the learning phase and that they were going to continue with the learning of a second set of items which also had to be retained. Then, the items of L2 were presented. Participants were again told that five SFMs were to be learned. Items were repeated in 12 blocks each presenting the items in a random order. We counterbalanced the assignment of SFMs to the two lists between participants.

We increased the retention interval by running a distractor phase. Participants worked on a Sudoku puzzle of medium difficulty printed on a sheet of paper. The experimenter interrupted them after $5 \mathrm{~min}$ so that they could move on to the test phase.

In the test phase, participants had to recall the items of both lists. Participants were instructed to enter the SFM in response to an exclamation mark appearing on the screen (cf. Fig. 1, middle section). As soon as the exclamation mark appeared, input could begin. After pressing four keys, it disappeared from the screen. It reappeared after $1 \mathrm{~s}$ signaling input of the next SFM. The test was divided by lists. Participants were instructed that they had to enter as many SFMs as a list comprised in each part of the test and were encouraged to guess if they were not able to remember the items with certainty. For the first part of the test, the forget group was asked to recall SFMs from the beginning of the experiment that had been designated as to-be-forgotten. Contrary to this previous instruction, they now were to try to remember the SFMs. The remember group was asked to recall the SFMs from the first part of the learning phase. For

\section{Learning}

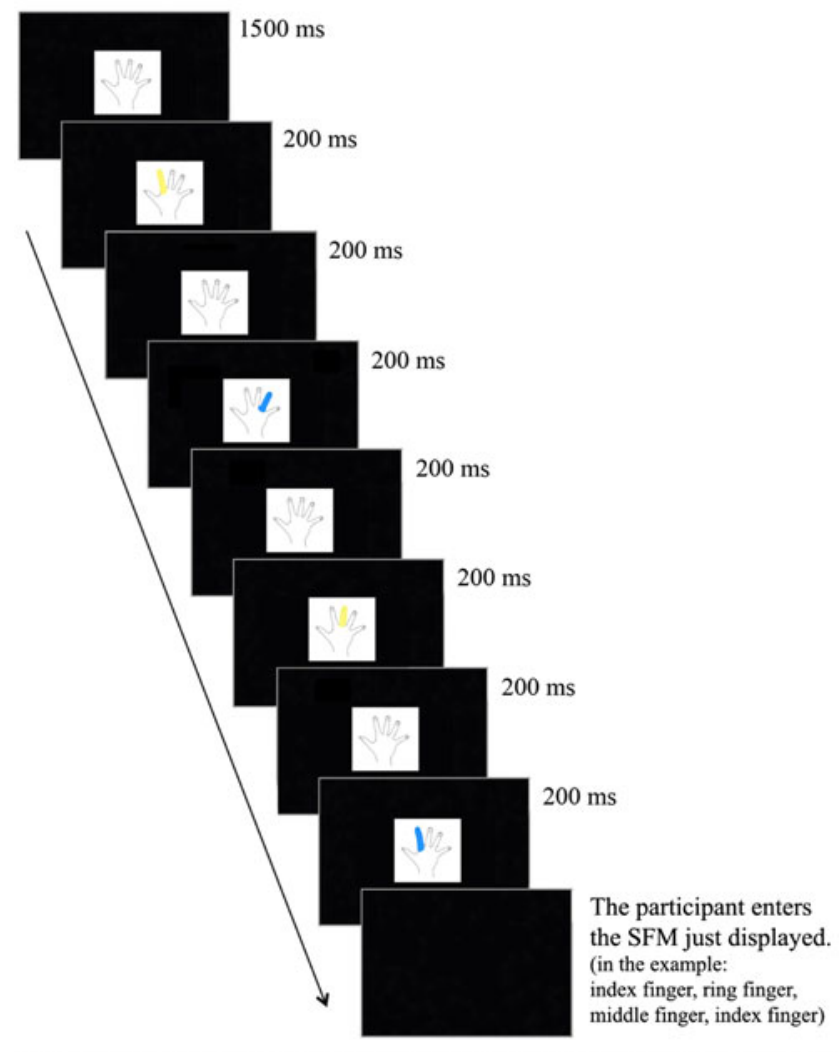

Test

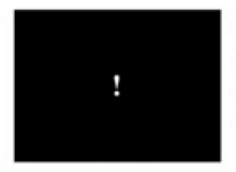

The participant enters a SFM. The exclamation point disappears after input. After $1000 \mathrm{~ms}$ a next exclamation point appears.

\section{Recall results}

\section{Experiment 1}

Experiment 2

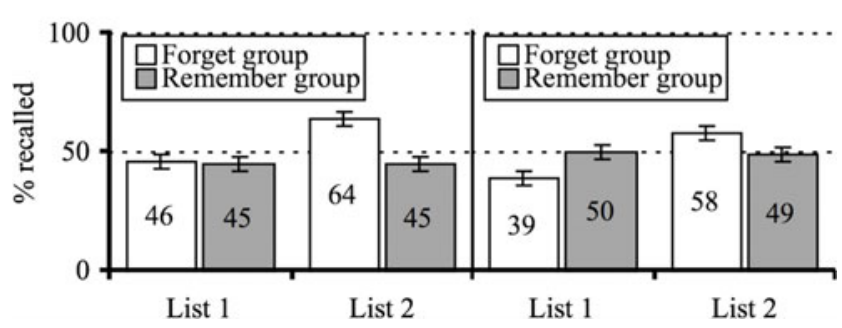

Fig. 1 The upper section depicts a trial in the learning phase. It starts with a drawing of the right hand. After 1,500 ms the first finger illuminates yellow for $200 \mathrm{~ms}$ followed by $200 \mathrm{~ms}$ of the uncolored drawing. Then the second finger flashes blue, the third finger yellow, and the fourth finger blue again in the same fashion. Subsequently, the hand display disappears and the participant can enter the sequential finger movement (SFM) just illustrated. The middle section depicts a trial in the test phase. Participants are supposed to enter a SFM as soon as an exclamation mark appears on the screen. The lower section shows recall results. The columns represent the percentages of recalled list- 1 and list- 2 items (i.e., items recalled relative to the total number of five items per list) in the forget group and in the remember group. Error bars depict standard error of the mean 
the second part of the test, participants were instructed to recall the SFMs from the second part of the learning phase. Participants were free to recall the SFMs of a list in any order they wished.

\section{Results}

Double input of SFMs was considered as incorrect recall, that is, when a SFM was entered twice it was counted as recalled only once. The forget group recalled significantly more SFMs from L2 than the remember group, $t(58)=2.83, p=.006$, indicating a benefit of directed forgetting (see Fig. 1, lower section). Intrusions from L1 (i.e., SFMs of L1 entered during the test for L2) did not differ significantly between groups, $|t|<$ 1 (forget group: $M=22 \%, S D=20 \%$; remember group: $M=$ $23 \%, S D=18 \%$ ).

The recall of $\mathrm{L} 1$ items did not differ significantly between the forget and the remember group, $|t|<1$. Intrusions from L2 (i.e., SFMs from L2 entered during the test for L1) did not differ significantly between groups, $t(58)=1.37, p=.177$ (forget group: $M=35 \%, S D=21 \%$; remember group: $M=$ $28 \%, S D=21 \%)$.

\section{Discussion}

The instruction to forget $\mathrm{L} 1$ enhanced recall of $\mathrm{L} 2$. The testing order requiring recall of $\mathrm{L} 2$ after recall of $\mathrm{L} 1$ suggests that this benefit resulted from better encoding of SFMs after receiving a forget instruction (Pastötter et al., 2012). This conclusion is further corroborated by the absence of a cost effect for L1. Obviously, the accessibility of L1 items did not differ between groups in the present experiment. Hence, any interference on the retrieval of L 2 items by L1 items was the same and, thus, cannot be the cause of the benefit effect.

However, the lack of a cost effect was unexpected. With regard to verbal materials, the cost effect has been proven to be quite robust, although certain conditions can abolish it, such as context reinstatement procedures (Sahakyan \& Kelley, 2002). We think that the task requirements in the learning phase here might have precluded processes that usually result in cost effects. These processes may comprise set segregation (Bjork, 1972), inhibition (Bjork, 1989), or an internal context change (Sahakyan \& Kelley, 2002). The task of repetitively entering SFMs all consisting of the same fingers and to be executed on the same keys in L1 and L2 could have hindered each of these processes. In a second experiment, we modified the procedure in order to facilitate the occurrence of a cost effect. We relied on modifications that could facilitate set segregation, inhibition, as well as an internal context change because there is still an ongoing debate in the literature on the extent to which these processes actually account for cost effects. The multifaceted evidence might suggest that all of them contribute to certain degrees.

\section{Experiment 2}

In Experiment 2, we aimed at improving the conditions for processes that resulted in costs of directed forgetting. We included a break of 3 min after L1. Instructions emphasized that participants ought to relax and prepare for the continuation of the learning phase (remember group) or, alternatively, for the start of the real experiment (forget group). In addition, different response keys were used for performing L1 and L2. We expected these modifications to potentially facilitate processes that have been suggested to account for cost effects, such as set segregation, inhibition, or an internal context change. Moreover, we increased the sample size, assuming that the size of a cost effect might still be relatively small.

\section{Method}

Participants and design One hundred and twenty undergraduate students at the University of Trier participated in the experiment. They either received course credit for their participation or were paid four Euro. The design was identical to Experiment 1.

Materials and procedure The same items were used as in Experiment 1. The procedure was also identical, except for two modifications. First, in the learning phase, the SFMs of L1 were performed on different response keys, 1, 2, and 3 of the numeric keypad on the right side of the keyboard, whereas the SFMs of L2 were again performed on the keys $V, B$, and $N$ that also served for entering the SFMs in the test phase. Second, there was a 3-min break between L1 and L2 in the learning phase. Participants were instructed to relax during this break to prepare and concentrate for the continuation of the experiment. Whereas the remember group was told that they were going to continue with the second part of the learning phase, the forget group was instructed to prepare for the start of the real experiment and to forget the thus far presented SFMs because they had only served the purpose of familiarizing participants with the set-up of the experiment.

\section{Results}

Indicating a cost effect of directed forgetting, the forget group recalled significantly fewer $\mathrm{L} 1$ items than the remember group, $t(118)=2.44, p=.016$. Intrusions from L2 (i.e., SFMs of L2 entered during the test for L1) did not differ significantly between groups, $t(118)=1.35, p=.181$ (forget group: $M=31 \%$, $S D=17 \%$; remember group: $M=26 \%, S D=19 \%$ ).

There was also a benefit of directed forgetting. The forget group recalled significantly more SFMs from L2 than the remember group, $t(118)=2.13, p=.035$. Intrusions 
from L1 (i.e., SFMs from L1 entered during the test for L2) did not differ significantly between groups, $|t|<1$ (forget group: $M=22 \%, S D=18 \%$; remember group: $M=25 \%$, $S D=17 \%$ ).

\section{Discussion}

Again, the instruction to forget L1 enhanced recall of L2. Thus, a benefit of directed forgetting was replicated. In addition, a cost effect for L1 occurred. Apparently, including a break in the learning phase between L1 and L2 and a change in response keys enabled processes that had been precluded in Experiment 1.

\section{Mediation analysis}

The two experiments provide converging evidence that the intention to forget just-learned motor sequences enhances subsequent encoding of motor sequences. Testing L2 after L1 should preclude interference by L1 to substantially impact the retrieval of L2 items (Pastötter et al., 2012). Moreover, the benefit effect was independent from the cost effect because it occurred in both experiments, whereas a cost effect only emerged in Experiment 2. Hence, the benefit effect did not depend on the accessibility of L1, but also occurred when L1 was as accessible in the forget group as in the remember group. However, there is another way to test the assumption that the benefit effect resulted from better encoding of L2 in the forget group. The accuracy at entering the SFMs in the learning phase can be considered an index of the encoding quality. Pastötter and Bäuml (2010) assume that the forget instruction abolishes memory load and inattentional encoding that results from the retention of $\mathrm{L} 1$ in the remember group. With regard to performing SFMs, lower memory load and better attention imply fewer errors, although accuracy might not be a pure measure of encoding quality, of course.

We examined whether encoding accuracy mediated the influence of the forget instruction on L2 recall. The number of correctly entered SFMs represented encoding accuracy. Data from both experiments were collapsed because we expected effect sizes to be medium to small. In particular the sample size of Experiment 1 alone would only have sufficed to detect large mediation effects (e.g., MacKinnon, Lockwood, Hoffman, West, \& Sheets, 2002). The encoding accuracy of L2 was significantly higher in the forget groups than in the remember groups, whereas encoding accuracy of L1 did not differ significantly between the forget and remember groups, $|t|<$ 1(see Fig. 2). L2 accuracy predicted L2 recall significantly. Including L2 accuracy as a second predictor in a regression analysis with L2 recall as the dependent variable reduced the impact of the predictor group, indicating a partial mediation. A Sobel test confirmed that the mediation was significant, $z=2.06, p=.039$. $^{1}$

\section{General discussion}

The intention to forget just-learned motor sequences enhanced the subsequent encoding of motor sequences. This finding matches recent dual-process models of directed forgetting (Pastötter \& Bäuml, 2010; Sahakyan \& Delaney, 2003). The benefit effect is not a mere byproduct of the cost effect but rather results from a separate source. Although reduced interference by L1 also can contribute to superior retrieval of L2 items in the forget group, the forget instruction already enhances encoding. In the present experiments, this impact was apparent from the occurrence of a benefit effect for L2 tested after L1, even in the absence of a cost effect. Moreover, the forget instruction increased encoding accuracy, which accounted for better recall of L2 items at test.

The measure of encoding accuracy, for the first time, provides direct evidence on the effect of directed forgetting on subsequent learning trials. Indeed, accuracy in performing the SFMs is not a pure measure of encoding quality, but it reflects more attentive learning in the forget group. This measure matches the reset-of-encoding assumption by Pastötter and Bäuml (2010) who posit that the forget instruction frees memory load and abolishes inattentional encoding. Previous evidence in favor of the reset-of-encoding hypothesis was the finding that benefit effects were restricted to the first-encoded L2 items but were absent for later items. In the present investigation, corresponding analyses face the limitation of ambiguous relative encoding positions because items were repeated in 12 blocks. Moreover, the relatively low number of only five items per list may preclude the emergence of a systematic decline of the benefit effect. Still, we performed analyses of item-specific recall probabilities as a function of serial positions in the first learning block. The interaction in a 2 (group) $\times$ 5 (learning position) ANOVA was not significant in Experiment $1, F(4,232)=1.75, p=.140$, or in Experiment $2, F(4,472)=1.80, p=.128$, indicating no decline of the

\footnotetext{
${ }^{1}$ In an additional mediation analysis we controlled for $\mathrm{L} 1$ recall by including it as a further predictor. The results were almost identical. Again, the encoding accuracy of L2 was significantly higher in the forget groups than in the remember groups, $\beta=.19, t(177)=2.57, p=.011$. L2 accuracy predicted L2 recall significantly, $\beta=.24, t(176)=3.75, p<.001$. Including L2 accuracy as a predictor in a regression analysis with L2 recall as the dependent variable reduced the impact of the predictor group from $\beta=.31, t(177)=4.67, p<.001$, to $\beta=.26, t(176)=4.04, p<.001$. A Sobel test confirmed that the mediation was significant, $z=2.12, p=.034$. In both experiments, mean L2 accuracy was higher in the forget group (Experiment 1: $89 \%$, Experiment 2: $89 \%$ ) than in the remember group (Experiment 1: $84 \%$, Experiment 2: $86 \%$ ).
} 

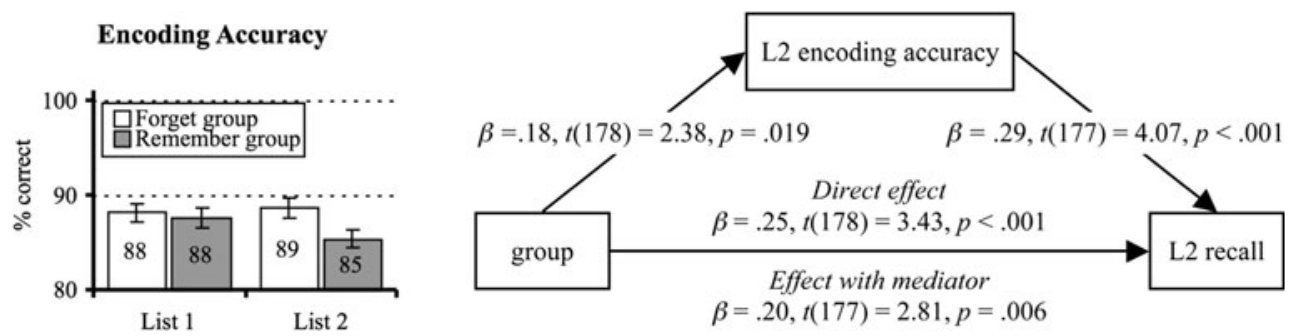

Fig. 2 The left section depicts the influence of experimental group on encoding accuracy (collapsed over experiments). The right section shows a mediation model with encoding accuracy for list 2 (L2) as a mediator of

directed-forgetting benefit with encoding position. We do not consider this particular null effect to speak against the reset-ofencoding hypothesis, given the mentioned limitations of these analyses here. However, the alternative assumption of a shift of encoding strategy in the forget group should be taken into account as a further explanation. Sahakyan and Delaney (2003) posit that the forget instruction triggers a shift to deeper semantic encoding of L2 with regard to verbal material. Although it is not clear how levels of processing may be distinguished with regard to motor sequences, it remains possible that a change to a somehow more efficient strategy contributed to the observed benefit.

The mediation of the recall benefit by encoding accuracy was significant but not complete, indicating that encoding accuracy did not reflect all aspects of encoding quality. Perhaps other measures, such as EEG or MRI, could capture more facets of encoding beside pure behavioral assessment. Still, the use of the novel item material of motor sequences already allowed for data from the learning phase that has never been available in studies with verbal materials before. Thus, broadening the paradigms of memory research and opening them to other realms of human behavior beyond words not only tests the relevance and applicability of insights from memory research to nonverbal domains, but also offers new ways for testing basic assumptions, enriching theoretical reasoning. Measures of encoding accuracy are not a priori restricted to non-verbal materials, however. For example, entering letter strings would also provide data that might indicate encoding quality. Including motor components in the learning of verbal material could prove to be a further way to examine encoding processes.

Motor action is unnecessarily neglected in many areas of current memory research. Although many phenomena are not restricted to the verbal domain but affect perceptual and motor memory representations as well, different modalities may involve different boundary conditions or unique factors determining organization and accessibility. Here, we found the benefit of directed forgetting to occur independently of costs. A cost effect emerged only in Experiment 2, whereas benefit effects emerged in both experiments. Indeed, the parameters determining the occurrence of cost effects for motor actions require further examination. It should be scrutinized whether cost effects depend on a distinct break separating L1 and L2. the influence of group on recall of items. Error bars depict standard error of the mean

Moreover, it is possible that either a 3-min period separating L1 and L2 or a change in response keys may suffice for a cost effect to emerge. The combination of both elements in Experiment 2 prohibits any speculation about their relative contribution. Perhaps both facilitate an internal context change. In contrast to verbal material, the motor sequences used in the present study might depend on a change in external features (such as different response keys) that also enables a change in the internal context. Without a change of external features, the uniform task requirements of performing SFMs with three fingers of the same hand in L1 as well as L2 may preclude an internal context change. The present findings may suggest that basic memory phenomena generalize to motor action, while at the same time pointing to modality-specific confines. Both aspects ought to be incorporated in theories of motor behavior as well as applications of memory theories. Perhaps one application derived from the present study could be the instructed forgetting of practice trials in sports or music exercises in order to benefit subsequent learning.

Sahakyan and Foster (2009; see also Earles \& Kersten, 2002) investigated list-method directed forgetting of action phrases that were either enacted by participants or merely read. They found that a cost effect emerged for both kinds of encoding, but a benefit effect only emerged for read but not enacted phrases. This finding might seem contradictory, at first glance, to the occurrence of benefit effects for motor actions here. However, the material differs crucially because action phrases are one kind of verbal material, whereas the used SFMs were free of semantic content, not verbally presented, and depicted only graphically. In contrast, enacting action phrases is a powerful way of deep semantic encoding. Even better encoding is hardly possible for enacted action phrases. Therefore, the lack of benefit effects for enacted action phrases supports the conclusion that benefits of directed forgetting arise from better encoding of L2.

Directed forgetting is immediately beneficial. It facilitates subsequent learning. Motor sequences benefit as do verbal materials, but they provide novel, more direct evidence on this basic mechanism.

Acknowledgments This research was supported by grant TE 891/3-1 of the German Research Council (DFG). 


\section{Appendix}

Items The assignment of the items designated A and B to list 1 and list 2 was counterbalanced between participants

\begin{tabular}{lllll}
\hline & First finger & Second finger & Third finger & Fourth finger \\
A & Index finger & Ring finger & Middle finger & Index finger \\
A & Index finger & Middle finger & Ring finger & Middle finger \\
A & Ring finger & Middle finger & Index finger & Ring finger \\
A & Ring finger & Index finger & Middle finger & Index finger \\
A & Middle finger & Index finger & Middle finger & Ring finger \\
B & Index finger & Ring finger & Middle finger & Ring finger \\
B & Index finger & Middle finger & Ring finger & Index finger \\
B & Ring finger & Middle finger & Index finger & Middle finger \\
B & Ring finger & Index finger & Middle finger & Ring finger \\
B & Middle finger & Index finger & Middle finger & Index finger \\
\hline
\end{tabular}

\section{References}

Ascoli, K. M., \& Schmidt, R. A. (1969). Proactive interference in shortterm motor retention. Journal of Motor Behavior, 1, 29-35. doi:10. 1080/00222895.1969.10734831

Bjork, R. A. (1972). Theoretical implications of directed forgetting. In A. W. Melton \& E. Martin (Eds.), Coding processes in human memory (pp. 217-235). Washington, DC: Winston.

Bjork, R. A. (1989). Retrieval inhibition as an adaptive mechanism in human memory. In H. L. Roediger III \& F. I. M. Craik (Eds.), Varieties of memory and consciousness: Essays in honour of Endel Tulving (pp. 309-330). Hillsdale, NJ: Erlbaum.

Brashers-Krug, T., Shadmehr, R., \& Bizzi, E. (1996). Consolidation in human motor memory. Nature, 382, 252-255. doi:10.1038/ $382252 \mathrm{a} 0$

Burwitz, L. (1974). Proactive interference and directed forgetting in short-term motor memory. Journal of Experimental Psychology, 102, 799-805. doi:10.1037/h0036361

Cothros, N., Köhler, S., Dickie, E. W., Mirsattari, S. M., \& Gribble, P. L. (2006). Proactive interference as a result of persisting neural representations of previously learned motor skills in primary motor cortex. Journal of Cognitive Neuroscience, 18, 2167-2176. doi:10. 1162/jocn.2006.18.12.2167

Earles, J. L., \& Kersten, A. W. (2002). Directed forgetting of actions by younger and older adults. Psychonomic Bulletin \& Review, 9, 383388. doi:10.3758/BF03196297

Irtel, H. (2007). PXLab: The Psychological Experiments Laboratory [online]. Version 2.1.11. Mannheim (Germany): University of Mannheim. Available from <http://www.pxlab.de $>$

MacKinnon, D. P., Lockwood, C. M., Hoffman, J. M., West, S. G., \& Sheets, V. (2002). A comparison of methods to test mediation and other intervening variable effects. Psychological Methods, 7, 83104. doi:10.1037/1082-989X.7.1.83

Panzer, S., Wilde, H., \& Shea, C. H. (2006). Learning of similar complex movement sequences: Proactive and retroactive effects on learning. Journal of Motor Behavior, 38, 60-70. doi:10.3200/JMBR.38.1.6070

Pastötter, B., \& Bäuml, K.-H. T. (2010). Amount of postcue encoding predicts amount of directed forgetting. Journal of Experimental Psychology: Learning, Memory, and Cognition, 36, 54-65. doi:10. 1037/a0017406

Pastötter, B., Kliegl, O., \& Bäuml, K.-H. T. (2012). List-method directed forgetting: The forget cue improves both encoding and retrieval of postcue information. Memory \& Cognition, 40, 861-873. doi:10. 3758/s13421-012-0206-4

Reppa, I., Worth, E. R., Greville, W. J., \& Saunders, J. (2013). The representation of response effector and response location in episodic memory for newly acquired actions: Evidence from retrievalinduced forgetting. Acta Psychologica, 143, 210-217. doi:10. 1016/j.actpsy.2013.03.007

Sahakyan, L., \& Delaney, P. F. (2003). Can encoding differences explain the benefits of directed forgetting in the list-method paradigm? Journal of Memory and Language, 48, 195-201. doi:10.1016/ S0749-596X(02)00524-7

Sahakyan, L., \& Foster, N. L. (2009). Intentional forgetting of actions: Comparison of list-method and item-method directed forgetting. Journal of Memory and Language, 61, 134-152. doi:10.1016/j. jml.2009.02.006

Sahakyan, L., \& Kelley, C. M. (2002). A contextual change account of the directed forgetting effect. Journal of Experimental Psychology: Learning, Memory, and Cognition, 28, 1064-1072. doi:10.1037/ 0278-7393.28.6.1064

Tempel, T., \& Frings, C. (2013). Resolving interference between body movements: Retrieval-induced forgetting of motor sequences. Journal of Experimental Psychology: Learning, Memory, and Cognition, 39, 1152-1161. doi:10.1037/a0030336

Tempel, T., \& Frings, C. (2014a). Interference within hands: Retrievalinduced forgetting of left and right hand movements. Acta Psychologica, 148, 1-5. doi:10.1016/j.actpsy.2014.01.003

Tempel, T., \& Frings, C. (2014b). Forgetting motor programmes: Retrieval dynamics in procedural memory. Memory, 22, 1116 1125. doi:10.1080/09658211.2013.871293

Tempel, T., \& Frings, C. (2015). Categorization by movement direction: Retrieval-induced forgetting of motor sequences grouped by motion features. Quarterly Journal of Experimental Psychology, 68, 473486. doi:10.1080/17470218.2014.945098

Tempel, T., Loran, I., \& Frings, C. (2015). Dancing your moves away: How memory retrieval shapes complex motor action. Journal of Experimental Psychology: Applied. doi:10.1037/xap0000052

Williams, I. D. (1971). The effects of practice trials and prior learning on motor memory. Journal of Motor Behavior, 3, 205-212. doi:10. 1080/00222895.1971.10734903

Wohldmann, E. L., Healy, A. F., \& Bourne, L. E., Jr. (2008). A mental practice superiority effect: Less retroactive interference and more transfer than physical practice. Journal Experimental Psychology: Learning, Memory, and Cognition, 34, 823-833. doi:10.1037/02787393.34.4.823 